\title{
Le traitement des varicocèles est toujours d'actualité
}

\author{
Alain JARDIN \\ Service d'Urologie, Hôpital Bicêtre, Le Kremlin Bicêtre
}

\section{INTRODUCTION}

Les varicocèles sont toujours sujet de débats....même leur féminité a été l'objet de controverse !

Depuis plusieurs siècles, chirurgiens et urologues débattent sur la nécessité de traiter les varicocèles de l'homme ainsi que sur la façon de les traiter. Depuis 50 ans, les andrologues s'interrogent sur les liens entre varicocèle et infertilité masculine.

C'est une heureuse coïncidence que le dernier numéro du dernier millénaire du Journal of Urology publie in extenso, dans sa rubrique, "mile stone in urology", l'article de W. Selby Tulloch qui montrait en 1955, à propos de trente cas, dont trois cas d'azoospermie, que le traitement du varicocèle pouvait améliorer la fertilité des hommes qui en étaient porteurs [18].

Les 2500 publications répertoriées dans le medline à ce jour, consacrées à ce sujet, n'ont cependant pas vraiment convaincu la Communauté Médicale de ce lien pourtant évident, ni de l'utilité pourtant certaine de bien traiter ceux qui en sont porteurs (qu'ils aient une varicocèle symptomatique ou qu'ils aient une altération de leur spermogramme).

Il est évident qu'avant de proposer une cure de varicocèle à un homme infertile, il est impératif d'avoir exploré toutes les autres causes d'infertilité (et elles sont nombreuses) mais on ne peut accepter de considérer, comme certains l'ont fait, que le traitement des varicocèles chez les hommes infertiles devait, comme tous les autres traitements conventionnels de l'infertilité masculine, être abandonné.

Enfin, chirurgiens et radiologistes «interventionnels» débattent de la meilleure technique à appliquer. Nous n'entrerons pas dans ce débat, même si, en tant que chirurgien, notre préférence va à la chirurgie «incisionnelle» de la varicocèle, malgré l'affirmation par Benjamin Bell en 1796 [1] que pour traiter la varicocèle le bistouri était un moyen cruel ....recommandé par les anciens!

\section{QUE PEUT ON ATTENDRE DE LA CHIRURGIE ?}

- Le traitement chirurgical doit guérir de sa varicocèle avec un minimum de complication et séquelle, l'homme qui en est porteur.

- En supprimant les conséquences de la varicocèle sur le testicule, la chirurgie peut espérer améliorer la fertilité de l'homme infertile qui en est porteur.

- De la même façon, elle peut espérer prévenir chez l'adolescent qui est porteur de varicocèle ses conséquences néfastes et en particulier un trouble de la spermatogenèse

\section{Guérir la varicocèle}

La varicocèle apparaît généralement à la puberté au moment du développement du testicule. La varicocèle existe chez 10 à $15 \%$ des hommes.

La varicocèle est une dilatation des veines du cordon spermatique. Elle siège pratiquement toujours à gauche pour des raisons anatomiques : la veine spermatique gauche est longue, faite en plusieurs points de son trajet d'un réseau veineux, elle se jette à angle droit dans la veine rénale gauche.

La varicocèle est une conséquence de l'orthostatisme. Le reflux de la veine rénale gauche vers la veine spermatique,

Correspondance : Pr A. JARDIN - Service d'Urologie. Hôpital Bicêtre.78, avenue du Général Leclerc. 94270 Le Kremlin Bicêtre - Tel 0145213771 - Fax 0145212170 Email : alain.jardin@bct.up-hop-paris.fr 
du le plus souvent à une incompétence valvulaire, est favorisé par l'hyperpression dans la veine rénale gauche. En position debout, la veine rénale gauche est comprimée entre aorte et artère mésentérique supérieure qui supporte «le poids de l'intestin».

$\mathrm{Si}$ on accepte que la varicocèle soit due à un reflux du sang de la veine rénale gauche vers le testicule, la suppression de toutes les voies veineuses pouvant relier cette même veine rénale aux veines drainant le testicule, doit entraîner la guérison de la varicocèle. Les techniques chirurgicales sont multiples mais ont pratiquement toutes la même ambition. Quelque soit la voie d'abord et le procédé chirurgical utilisé le but est pratiquement toujours d'interrompre le courant veineux entre la veine rénale et le testicule.

L'utilisation du microscope recommandé par certains auteurs [17] ne peut être proposée systématiquement que si on veut chaque fois préserver l'artère spermatique ce qui nous parait discutable.

\section{a) Chirurgie sous coelioscopie}

La cure de la varicocèle a été une des premières interventions urologiques a être faite sous laparoscopie. De technique relativement aisée, elle ne peut prétendre avoir une vue aussi complète que la chirurgie ouverte de l'espace rétropéritonéal où cheminent les vaisseaux spermatiques. Elle ne semble pas apporter un bénéfice suffisant pour détrôner la chirurgie classique [3].

\section{b) Chirurgie incisionnelle}

Depuis plusieurs siècles, les techniques de cure de la varicocèle décrites ont été très nombreuses. En fait, la chirurgie par voie scrotale est pratiquement abandonnée et seules persistent les interventions menées par voie inguinale et les interventions menées par voie supra inguinale. Un chapitre de technique chirurgicale de la varicocèle ne peut omettre la contribution d'Ivanissevitch $[6,7]$. La plupart des chirurgiens s'accordent pour réaliser l'interruption du courant veineux spermatique dans sa totalité.

Les avis divergent sur l'importance ou non de conserver l'artère spermatique [17]. Il nous parait logique d'aborder les vaisseaux spermatiques à l'endroit où une veine spermatique est le mieux individualisée c'est-à-dire la région iliaque. A ce niveau l'intervention respecte obligatoirement les deux autres artères du testicule. Aussi le sacrifice de l'artère spermatique est-il pratiquement toujours sans conséquence [20].

Dans notre pratique [8] l'intervention se déroule de la façon suivante :

- le patient est installé en décubitus dorsal sur un plateau chirurgical permettant des radiographies.

- incision cutanée de 7 à $10 \mathrm{~cm}$ selon l'adiposité du patient. incision horizontale iliaque à égale distance de l'ombilic et de l'épine iliaque antérosupérieure.

- incision oblique de l'aponévrose du grand oblique.

- dissociation du petit oblique à $1 \mathrm{~cm}$ du bord externe du grand droit.

- incision de l'aponévrose du transverse.

- décollement et refoulement du péritoine où sont accolés les vaisseaux spermatiques. (Il est donc important de rester près du péritoine et non pas trop près de la paroi, plan qui mènerait en arrière du muscle psoas). Le péritoine refoulé, les veines spermatiques sont aisément reconnaissables et ne peuvent être confondues.

- parfois (moins de $10 \%$ des cas) l'artère spermatique est bien visible, isolée du reste du paquet spermatique. Elle est alors repérée et sera conservée.

- dans les autres cas, la veine spermatique ou la plus grosse des veines rencontrées est repérée et soulevée par deux fils.

- elle est incisée sur la moitié de sa circonférence.

Un cathéter adapté à la taille de la veine est mis en place dans le bout d'aval, un autre dans le bout d'amont. Chaque cathéter est maintenu en place par ligature des deux fils mis en place précédemment. $15 \mathrm{cc}$ de produit de contraste sont injectés dans chacun des cathéters sans presser.

Ces radiographies permettent :

- de visualiser la totalité de la veine spermatique et ses branches et la veine rénale.

- de mettre en évidence les anastomoses gauche, droite, sus pubiennes expliquant quelques varicocèles droits associés.

- surtout de visualiser des anastomoses et de mettre en évidence d'autres veines spermatiques ayant des trajets aberrants ; négliger de tels vaisseaux conduit à la récidive [12].

La lecture des radiographies dicte la poursuite de l'intervention :

- veine unique : pas de collatérale : repérage de l'artère qui ne sera préservée que si elle n'est pas accompagnée de petites veines en échelle indissociables de l'artère.

- veines multiples avec anastomoses : recherche de collatérales anormales et ligature de tous les éléments vasculaires depuis l'uretère, repéré, jusqu'à la paroi. Les vaisseaux spermatiques sont généralement réséqués sur 7 ou $8 \mathrm{~cm}$ avec ligature faite au fil résorbable (vicryl 3-0).

Après vérification de l'hémostase, la paroi est refermée.

La cure chirurgicale du varicocèle est un geste simple réglé. 
Ce geste est parfaitement réalisable en chirurgie ambulatoire.

Les complications sont exceptionnelles :

- La principale «complication» est représentée par la récidive qui peut survenir immédiatement si une branche se jetant dans la veine rénale gauche a été laissée en place ou plus tardivement (quelques semaines ou mois) si des petites veines collatérales ont été oubliées et à travers lesquelles se reconstitue un conduit veineux alimentant le varicocèle à partir du sang veineux rénal gauche.

- Le taux de récidive (1 à $15 \%$ ) est minimisé par la pratique de radiographie per opératoire.

- L'hydrocèle est une complication fréquente de la cure chirurgicale du varicocèle ( $8 \%$ dans notre expérience). Elle peut survenir plusieurs années après la cure de varicocèle. Sa pathogénie n'a jamais été parfaitement établie. Peut être le respect de lymphatiques que permet l'emploi du microscope peut-il avoir dans ce but un certain intérêt.

- L'atrophie testiculaire est tout à fait exceptionnelle (quelques cas isolés rapportés) en rapport avec des anomalies anatomiques artérielles, la crainte de cette complication a pu justifier pour certains le recours systématique à la microchirurgie (ceci ne nous parait pas justifié).

\section{Améliorer la fertilité de l'homme qui en est porteur}

Dans les années 50, la relation entre varicocèle et infertilité masculine a été mise en évidence.

- La fréquence des anomalies du spermogramme chez les porteurs de varicocèle n'est pas parfaitement établie (supérieure à $60 \%$ ).

- Dans une consultation d'hommes infertiles on retrouve $35 \%$ de porteurs de varicocèle [19]. Les mécanismes expliquant l'infertilité de la varicocèle sont encore discutés. Ils font l'objet de mises au point dans le numéro d'ANDROLOGIE de mars 2002.

Le volume du testicule gauche est diminué chez plus de la moitié des porteurs de varicocèle. Le testicule qui augmente de volume à la puberté atteint sa taille définitive environ à l'âge de 20 ans. La varicocèle développée lors de la puberté peut gêner la croissance normale du testicule jusqu'à sa taille définitive.

Enfin il est vraisemblable que souvent la varicocèle intervienne comme un cofacteur dans le déterminisme des troubles de la spermatogénèse. Aucun test n'est disponible pour déterminer chez un homme donné si la varicocèle est la seule cause de son infertilité. Il est donc important, du fait de la grande fréquence de cette pathologie chez un homme infertile et porteur de varicocèle, de rechercher un autre facteur d'infertilité (infection génitale, anomalie prostato-séminale, tabagisme, etc...). L'aggravation des anomalies de la spermatogenèse par le tabagisme chez les porteurs de varicocèle en est un exemple démonstratif [16].

Le caractère multifactoriel de l'étiologie de l'infertilité masculine rend compte des divergences parfois importantes des résultats publiés concernant les traitements des causes d'infertilité masculine.

Il est impossible de prévoir pour un couple donné le nombre de chances de succès en terme de grossesse de la chirurgie de la varicocèle de l'homme. Les résultats de la littérature font état d'un taux de grossesse après cure de varicocèle variant entre 7 et $75 \%$ (!). La moyenne des publications se situe autour de 25 à $30 \%$ de grossesses menées à terme, dans notre expérience elle est de $24 \%$. Ces chiffres sont loin d'être «décourageants».

Il parait donc raisonnable de conserver à la chirurgie de la varicocèle la place non négligeable qu'elle mérite.

Mais depuis près de 50 ans, la réalité de l'amélioration de la fertilité par la cure de la varicocèle a été contestée. La cure de la varicocèle, chez l'homme infertile a connu deux grandes périodes de contestation :

1. 1979 avec l'article de S. Nilsson [15] qui, à partir d'une étude randomisée comparant le nombre de grossesses obtenues chez des patients opérés et non opérés, montrait qu'il y avait plus de grossesses chez les non opérés que chez les opérés.

Plusieurs études contradictoires ont été publiées depuis [13, 14] et les arguments en faveur du traitement de la varicocèle ont primé. Ils ont été résumés dans l'article de Hargreave paru dans Human Reproduction en 1995 [5]. Cet article plein de sagesse aurait pu clore le débat [5] : il n'en fut rien. Il est difficile de convaincre !

2. 1992 avec la naissance de l'ICSI, les troubles de la spermatogenèse en rapport avec la varicocèle n'ont pas échappé aux excès de l'ICSI, résumés dans l'article de Van Sterteghem paru dans Human Reproduction en 1998 et qui écrivait : "les traitements conventionnels des facteurs masculins d'infertilité ont très peu de valeur, ils ont été révisés et abandonnés". (sic !) [2].

Comme toujours le bon sens ne fait pas bon ménage avec les attitudes excessives.

Le traitement chirurgical de la varicocèle de l'homme infertile a encore des indications à l'ère de l'I.C.S.I. Si l'I.C.S.I. a, de toute évidence, transformé le pronostic de l'infertilité masculine, ses inconvénients ne doivent pas être oubliés :

- nécessité d'une stimulation hormonale de la femme ;

- ponction ovarienne ; 
- risque de grossesses multiples ;

- gestion d'éventuels embryons surnuméraires à laquelle les couples sont souvent mal préparés ;

- évaluation encore limitée de la qualité du conceptus.

Il nous paraît peu admissible (bien que nous l'ayions constaté récemment) de proposer une F.I.V. voir une I.C.S.I. à un couple de 25 ans dont l'homme ayant au spermogramme une oligo-asthéno-tératospermie marquée était porteur d'une grosse varicocèle et fumait 30 cigarettes par jour. Il faut savoir expliquer et résister à la pression des couples et à celle de ceux qui en ont la charge.

La varicocèle de l'adulte de plus de 20 ans ne souffrant d'aucun symptôme et ayant un spermogramme normal ne justifie pas à nos yeux de geste chirurgical. Cependant si un tel homme n'a pas encore procréé, il semble justifié de proposer une autoconservation de sperme, une altération progressive des spermatozoïdes étant possible.

En cas d'altération du spermogramme, la cure de la varicocèle nous parait justifiée sous condition :

- d'avoir traité toute autre cause d'infertilité retrouvée ,

- d'obtenir l'arrêt d'une intoxication tabagique ,

- d'avoir recherché et traité une anomalie gynécologique chez la partenaire.

Ce schéma a en fait été modifié par les succès obtenus par procréation médicalement assistée et tout spécialement l'ICSI. Si la femme a plus de 35 ans, il peut être justifié de procéder d'emblée à une PMA si le délai d'attente d'une grossesse est supérieur à 2 ans.

Cet âge peut être abaissé s'il existe chez la femme des anomalies gynécologiques en particulier tubaires.

\section{Prévenir l'infertilité}

L'âge de la procréation décidée par les couples étant de plus en plus avancé, il paraît raisonnable de proposer à un adolescent ou à un adulte jeune porteur d'une varicocèle, ayant un retentissement sur le testicule, d'être opéré.

On peut ainsi espérer prévenir les conséquences néfastes de la varicocèle sur la spermatogénèse.

La varicocèle de l'adolescent a fait l'objet de nombreuses publications ces dernières années $[4,10,11]$. Il semble y avoir un effet délétère sur le testicule d'autant plus important que la varicocèle a un grade élevé [9].

Des études avec un long suivi sont encore nécessaires pour avoir une opinion définitive devant ce problème important de santé publique. En effet une attitude systématique d'indication chirurgicale chez l'adolescent reviendrait à opérer de varicocèles environ $10 \%$ de la population masculine.
Notre attitude à ce jour est la suivante :

- grosse varicocèle gênante : cure chirurgicale

- varicocèle non gênante :

- si les deux testicules sont symétriques : surveillance annuelle ;

- s'il y a une asymétrie testiculaire : cure chirurgicale.

Le spermogramme avant 18 ans peut être d'interprétation difficile, les normes du spermogramme avant cet âge n'étant pas parfaitement établies et variables d'un individu à l'autre.

$\mathrm{Si}$, à 20 ans, le spermogramme est normal et qu'il est décidé de ne pas opérer la varicocèle, il nous paraît prudent de conseiller une conservation du sperme.

\section{CONCLUSIONS}

Il n'est pas question de faire de la chirurgie de la varicocèle le traitement de l'infertilité masculine mais lorsqu'on peut donner à un couple jeune, par un geste chirurgical peu invasif, la possibilité de procréer naturellement, en évitant les aléas de la PMA in vitro, il ne faut pas s'en priver.

\section{RÉFÉRENCES}

1. BELL B. : De la varicocèle, de la cirsocèle, de la spermatocèle, et de la pneumatocèle. Cours complet de chirurgie théorique et pratique. 1796. Traduit de l'anglais par Ed. Bosquillon. Tome 1, Ch $8,281$.

2. DEWROEY P., VAN STEIRTEGHEM A. : Do we treat the male or his gamete ? Hum. Reprod., 1998, 13, suppl. 1 : 178-185.

3. ENQUIST E., STEIN B.S., SIGMAN M. : Laparoscopic versus subinguinal varico-celectomy : a comparative study. Fertil. Steril., 1994, 61 : 1092-1096.

4. FONTAINE E., JARDIN A. : La varicocèle de l'adolescent. Progr. Urol., 2001, 11 : 729-732.

5. HARGREAVE T.B. : Debate on the pros and cons of varicocele treatment in favour of varicocele treatment. Human Reprod., 1995, 10, suppl. $1: 151-157$.

6. IVANISSEVITCH O. : Left varicocele due to reflux. Experience with 4.470 operative cases in forty two years. J. Int. Coll. Surg., $1960,34: 742$.

7. IVANISSEVICH O., GREGORINI H. : Una nueva operacion para curar el varicocele. Semana medicin Buenos Aires. 1918, 25: 575 .

8. JARDIN A. : Varicocele. In : Glenn's Urologic Surgery. 5th Ed., Lippincott-Raven-USA, 1998 : 507-514.

9. KASS E.J. : Adolescent varicocele. Pediatr. Clin. North Am., $2001,48: 1559-1569$.

10. KASS E.J., STORK B.R., STEINERT B.W. : Varicocele in adolescence induces left and right testicular volume loss. Br. J. Urol. Int., 2001, $87:$ 499-501.

11. LAVEN J.D., HAANS I.C.F., MALI W.P., EGBERT R.V., WEN- 
SING C.J., EIMERS J.M. : Effects of varicocele treatment in adolescents : a randomized study. Fertil. Steril., 1992, 58 : 756-762.

12. LENZ M., HOF N., KERSTING-SOMMERHOFF B., BAUTZ W. : Anatomic variants of the spermatic vein, importance for percutaneous sclerotherapy of idiopathic varicocele. Radiology, 1996, $198: 425-431$.

13. MADGAR I., WEISSENBERG R., LUNENFELD B., KARASIK A., GOLDWASSER B. : Controlled trial of high spermatic vein ligation for varicocele in infertile men. Fertil. Steril., 1995, $63: 120-124$.

14. NIESCHALG E., HERTLE L., FISCHEDICK A., BEHRE H.M.: Treatment of varicocele : counselling as effective as occlusion of the vena spermatica. Hum. Reprod., 1995, $10: 347-353$.

15. NILSSON S., EDVINSON A., NILSSON B. : Improvement in semen and pregnancy rate after ligation and division of the internal spermatic vein : fact or fiction ? Br. J. Urol., 1979, 51 : 591 596.

16. RENG B.C.H. : The cofactor effect : varicocele and infertility. Fertil. Steril., 1990, 54 : 143-148.

17. SILBER S.J. : Microsurgical aspects of varicocele. Fertil. Steril., $1979,31: 230-232$.

18. TULLOCH W.S. (1955) : Mile stone in urology : Varicocele in subfertility - Results of treatment. J. Urol., 2001, 166 : 20322034.

19. W.H.O. : The influence of varicocele on parameters of fertility in a large group of men presenting to infertility clinics. Fertil. Steril., 1992, $57: 1289-1293$.

20. YAMAMMOTO M., TSUJI Y., OHMURA M., HIBI H., MIYAKE K. : Comparison of artery-ligating and artery-preserving varicocelectomy : effect on post-operative spermatogenesis. Andrologia, 1995, $27: 37-40$. 